\title{
Brain Tumor Detection using Image Processing
}

\author{
N. Sravanthi ${ }^{1}$, Nagari Swetha ${ }^{2}$, Poreddy Rupa Devi ${ }^{3}$, Siliveru Rachana ${ }^{4}$, Suwarna Gothane ${ }^{5}$, N. Sateesh ${ }^{6}$ \\ ${ }^{1}$ Assistant Professor, Department of Computer Science and Engineering, CMR Technical Campus, Hyderabad, Telangana, India \\ ${ }^{2-4}$ B. Tech Scholar, Department of Computer Science and Engineering, CMR Technical Campus, Hyderabad, Telangana, India \\ ${ }^{5}$ Associate Professor, Department of Computer Science and Engineering, CMR Technical Campus, Hyderabad, Telangana, India \\ ${ }^{6}$ Professor and HoD, Department of ME, Gokaraju Rangaraju. Institute of Engineering and Technology, Hyderabad, Telangana, India
}

\section{Article Info}

Volume 7, Issue 3

Page Number: 348-352

\section{Publication Issue :}

May-June-2021

\section{Article History}

Accepted : 20 May 2021

Published : 29 May 2021

\section{ABSTRACT}

It is very difficult for doctors to detect a brain tumor at an early stage. MRI images are more susceptible to noise and other environmental disturbances. Therefore, it becomes difficult for doctors to determine the tumor and its causes. So, we came up with a system in which the system will detect a brain tumor from images. Here we are converting an image to a grayscale image. We apply filters to the image to remove noise and other environmental clutter from the image. The system will process the selected image using preprocessing steps. At the same time, different algorithms are used to detect the tumor from the image. But the edges of the image will not be sharp in the early stages of a brain tumor. So here we are applying image segmentation to the image to detect the edges of the images. We have proposed an image segmentation process and a variety of image filtering techniques to obtain image characteristics. Through this entire process, accuracy can be improved. This system is implemented in the Matlab.

Keywords : Brain Tumor, classification, Segmentation.

\section{INTRODUCTION}

This work proposes two different methodologies for segmenting a tumor on an MRI image and determining the type of tumor. For this, one segmentation technique and one clustering were implemented. Among the various brain problems, the most common and life-threatening problem these days is a brain tumor. About 11,000 people are diagnosed with a brain tumor every year [1]. Annually, about 1 lakh of 50 thousand cancer patients are affected by a brain tumor [2]. A render chain in which the image is preprocessed to remove noise. A brain tumor develops due to unusual cell growth in the brain [3].

\section{LITERATURE REVIEW}

Rasel Ahmmed Anirban Sen Swkshar, Md. Foisal Hossain, Md. Abdur Rafiq [4] proposed method which include stages like image pre-processing, segmentation, feature extraction, SVM classification and tumor stage classification using Artificial Neural Network (ANN). Key image processing techniques for brain MRI image segmentation is classified as SVM, FCM. Swapnil R. Telrandhe, et. al [5] Proposed tumor detection inside which Segmentation separates an image into parts of regions or objects. In this it has to segment the item from the background to browse the image properly and classify the content of the image strictly. During this framework, edge detection 
is a vital tool for image segmentation. In this paper their effort was made to study the performance of most commonly used edge detection techniques for image segmentation and additionally the comparison of these techniques was carried out with an experiment.

Preliminary results show that our approach has achieved good segmentation results. Also this approach was reduces a large quantity of calculation.

\section{EXISTING SYSTEM}

In existing system, brain cancer was detected using image processing techniques. Initially a brain CT scan image is acquired and the pre-processing techniques are applied on it. Then pre-processed image is segmented. In these systems, the techniques used in the detection of the brain cancer were confined to segmentation.

These approaches are also suffered from certain issues which can be removed by improving the technology used in it. Furthermore, all the work has done only for detecting whether it's cancerous or not but none detected to which stage does the cancer belong.

\section{DISADVANTAGES}

Many systems are confined only to segmentation.

$>$ They suffer from certain technology issues.

Some of the systems work only on cancerous image.

Staging is not done.

$>$ Less accuracy.

Area of the tumor isn't computed.

\section{PROPOSED SYSTEM ARCHITECTURE}

The proposed method for detecting a brain tumor includes three diagnostic tasks: pre-processing, segmentation, and feature extraction. At a later stage, we calculate and classify the area based on this. As mentioned above, the obtained CT image is preprocessed. The preprocessed image is segmented and later we extract features from the segmented image. Finally, we classify the image based on the extracted features. In this project, we described our goal in two parts, the first half concerns the detection of a brain tumor, that is, the presence of a tumor on the provided MRI. The other part, that is, the second part, contains the classification of the tumor. In general, the diagram of our process. The input images will go through various stages, which can be summarized as follows, which are shown in Figure 1.

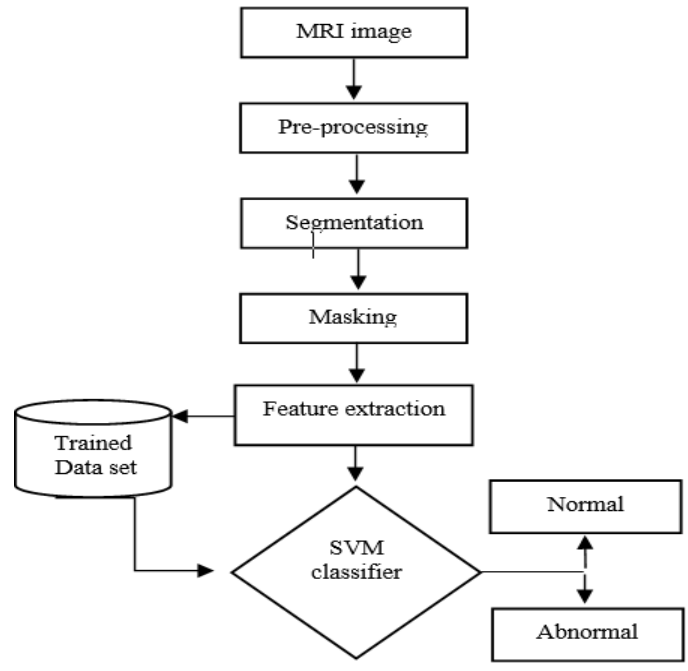

Fig. 1: Brain tumor detection steps.

The first step in brain tumor detection involves taking the input of the MRI image as shown above. The MRI image will undergo preprocessing and segmentation techniques. The aim of preprocessing is make to improvements to the image data that enhance the image features which are important for further processing. Image segmentation is the process of dividing a digital image into multiple segments that will be helpful in further Processes. In feature extraction, all the features from the input images will be extracted and then the acquired features will be compared with the trained data set. In the end, after comparing with the trained data set the output will tell whether the tumor is present or not. If there exists the tumor the output will be abnormal or else the output will be as normal. 


\subsection{MRI images of the brain}

This is the first step of the proposed system. The resulting MRI images may not be of very good quality for analysis. Images can be noisy, blurry, low-contrast. The area of interest can be difficult to extract [14]. Here, grayscale MRI images are provided as input to the system.

\subsection{Pre-Processing}

This is the initial processing of data in order to prepare them for primary processing or further analysis. The preprocessing phase of our project mainly includes those operations that are usually necessary before the target analysis and extraction of the necessary data and usually geometric corrections of the original image. These improvements include correcting information for jaggedness and unwanted noise in an area, removing an image of a non-brain element, and transforming the data so that it reflects correctly in the original image. The first preprocessing step is to transform this input MRI image into a suitable form with which further work can be done.

\subsection{Feature Extraction}

It is the process by which certain features of interest in an image are detected and presented for further processing. This is an important step in most computer vision and imaging solutions. Based on the results obtained during the extraction of signs, the tumor is classified. When extracting, certain parameters are taken into account: size, shape, composition, image location. This step extracts the features of the given input image. Based on these characteristics, the image is analyzed and the area of the tumor is determined. Figure 2 below shows the output of the MRI image prior to the feature extraction phase of the project.

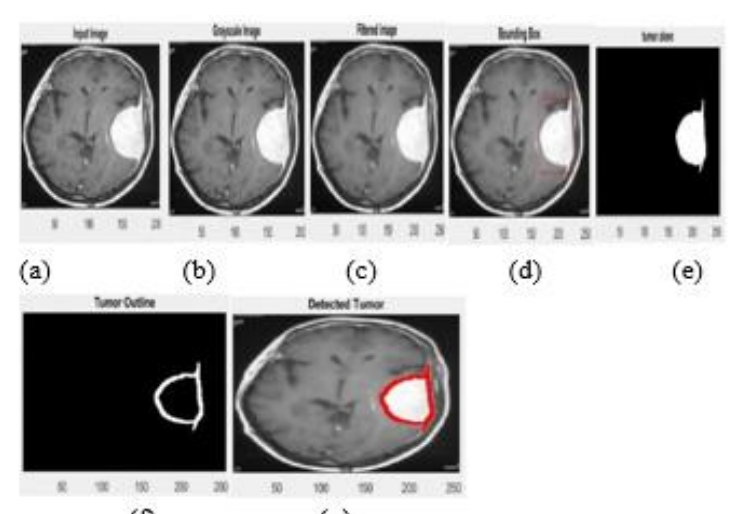

(f)

(g)

Fig. 2: (a) Input image, (b) Grayscale image, (c) Filtered image, (d) Bounding box, (e) Tumor alone image, (f) Tumor outline, (g) Detected tumor.

\subsection{Segmentation}

Segmentation is a method of breaking up an image into smaller pieces. Performed to facilitate analysis. Segmentation in this project refers to the method of dividing an image into many segments, however, the greatest difficulties in segmentation are related to the degree of the image, and images are also not inherited in a continuous area, as on X-ray film, or in a separate house, as in MRI. In 2D individual images, the placement of each action is called an element, and in $3 \mathrm{D}$ images it is called a voxel.

When the restriction that regions connect is removed, the defining sets are called pixel classifications, and therefore the sets themselves are called classes.

To address this problem, we used the foremost reliable segmentation techniques, which are helper vector machines and self-organizing maps, to see if there's a tumor on the input MRI image.

The support vector machine (SVM) approach is considered a good candidate because of its high generalization performance, especially when the size of the function space is very large. SVM uses the following idea. The SVM takes operational images as input and gives the accuracy of a neural network with manual options in a purely handwriting 
recognition task. Those training points for which the equality of the dividing plane is satisfied, those that lie on one of the hyperplanes $(\mathrm{H} 1, \mathrm{H} 2)$ and the removal of which will change the found solution, are called support vectors (SV).

\subsection{Image Analysis}

After determining the type of tumor, an image analysis is performed to identify a brain tumor.

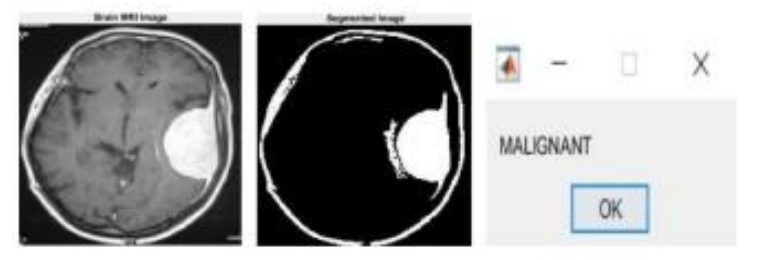

Fig. 3: Showing the result of the taken MRI image and providing the classification of the type of tumor.

\section{ADVANTAGES}

In our proposed system, we classified the image based upon the area of the tumor.

$>$ Staging is done.

$>$ More accuracy.

Area of the tumor is computed.

\section{SYSTEM REQUIREMENTS}

\subsection{SOFTWARE REQUIREMENTS:}

$>$ Operating system : Windows7 \& above versions

$>$ Coding Language :MATLAB

> 5.2 HARDWARE REQUIREMENTS:

$>$ Processor : Intel i3 and above.

$>$ RAM : $4 \mathrm{~GB}$ and higher.

$>$ HardDisk : 500GB minimum.

\section{IMPLEMENTATION}

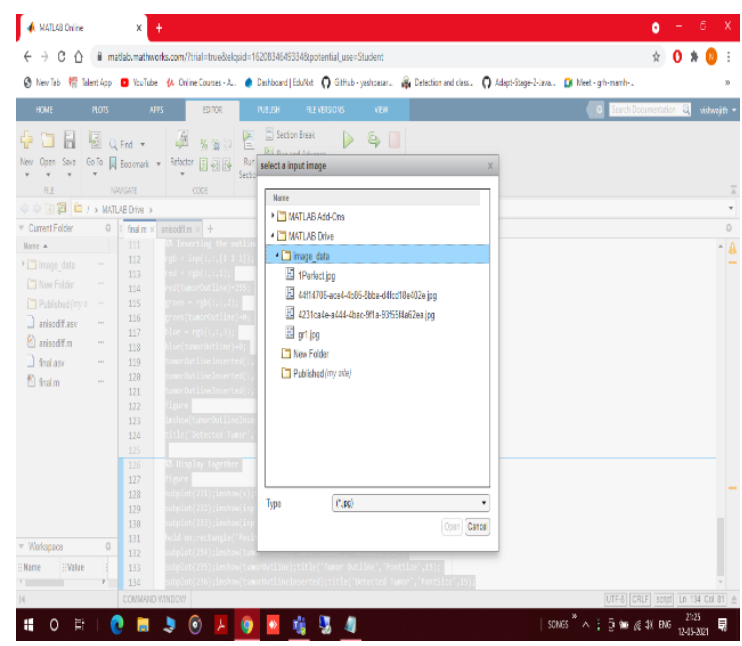

Fig. 4: Selecting the MRI for detection of tumor.

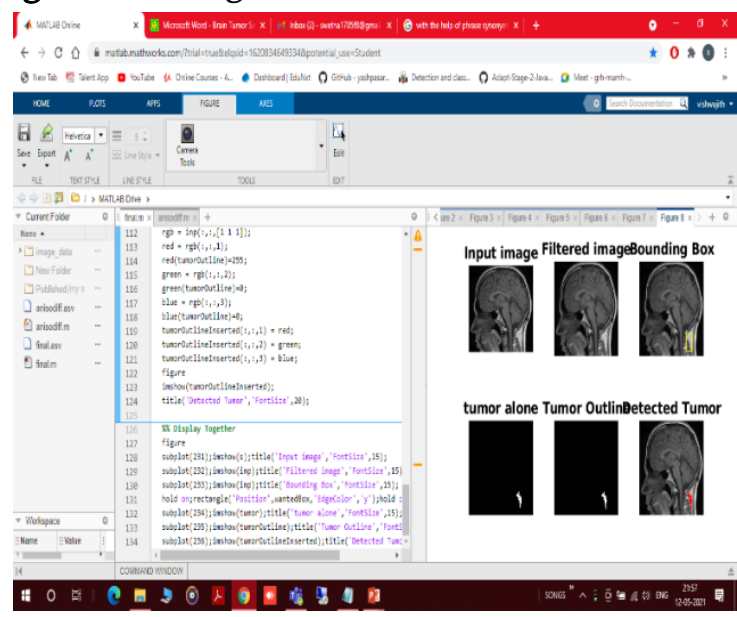

Fig. 5: Showing the output of detected tumor.

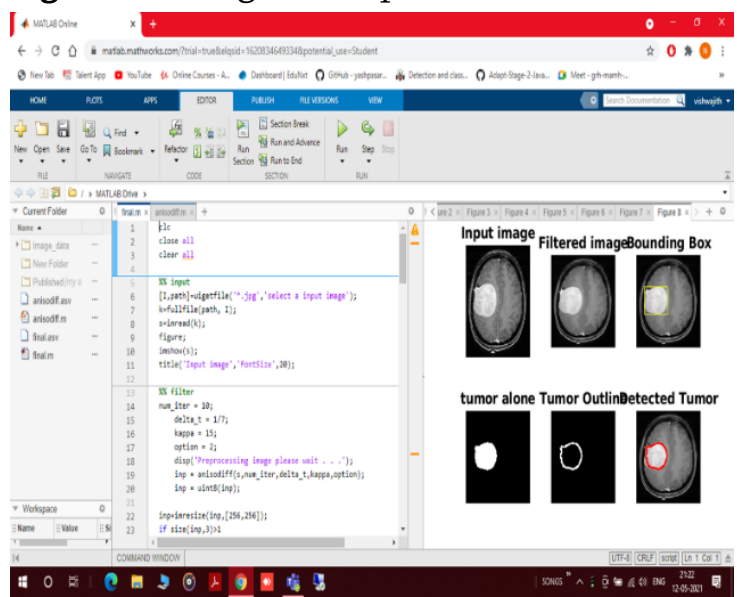

Fig. 6: Result of detected tumor which is highlighted with red colour around tumor.

\section{CONCLUSION AND FUTURE SCOPE}

It is a system that can be used to segment MRI images of the brain to detect and identify a brain tumor. This 
system determines the size of the tumor with maximum precision. In this project, we have automated the procedure for diagnosing a brain tumor using image processing. In addition to several existing methodologies for segmentation and detection of brain tumors for MRI images of the brain, our project has proven to provide an overall accuracy of up to $97 \%$. The preprocessing includes operations such as wavelet-based methods have already been discussed. Enhancement and filtering are important because sharpening edges, enhancing, removing noise, and removing unwanted background improves image quality as well as detection. Among the various filtering methods: reduces noise; improve image quality and improve computational efficiency compared to other filtering methods. Following the improved image quality and noise reduction discussed here, the brain tumor segmentation methodology based on MRI images of the brain was used. Classification-based segmentation accurately segments the tumor and yields reasonable results for a large set of information, however, unwanted behavior can occur if the category is not represented in the training data. These classification methods can first determine if a tumor is present or not, and if there is one, they can determine if a tumor is present or not.

The possibilities for detecting a brain tumor in the future are that if we get a three-dimensional image of the brain with the tumor, then we can also estimate the type of tumor as well as the stage of the tumor.

\section{REFERENCES}

[1]. Prof. "What is a brain tumor? The Brin Tumor Chrity."Online.Availble:https://www.thebtintu $\mathrm{m} \quad$ orchrity.org/understanding-brintumours/ symptomsnd-informtion/what-is braintumour/.

[2]. "Brain Tumors Clssifications,Symptoms,Dignosis and Treatments. "Online Available:
https://www.aans.org/Patients/Neurosur giclConditions-nd-Treatments/BrinTumors.

[3]. Riddhi S. Kapse1, Dr. S. S. Salankar2 and Madhuri.B abar3 "Literature Survey on Detection of Brain Tumor from MRI Images" IOSR Journal of Electronics and Communication Engineering (IOSR-JECE) e-ISSN: 2278-2834, pISSN: 2278-8735. Volume 10, Issue 1, Ver. II (Jan-Feb.2015), PP 80-86.

[4]. Rasel Ahmmed Anirban Sen Swkshar,Md.Foisal ,and Md.Abdur Rafiq"Classification of Tumors and It Stages in Brain MRI Using Support Vector Machine and Artificial Neural Network"in International Conference on Eletrical,Computer and Communication Engineering(ECCE),Cox's Bazar,Bangladesh,February 16-18,2017.

[5]. Sijbers J et al (1998), "Estimation of the noise in magnitude MR images”, Magn Reson Imaging 16(1):87- 90.

[6]. MathWorks

(https://in.mathworks.com/help/wavelet/ug/lifti ng-method-forconstructingwavelets.html).

\section{Cite this article as :}

N. Sravanthi, Nagari Swetha, Poreddy Rupa Devi, Siliveru Rachana, Suwarna Gothane, N. Sateesh, "Brain Tumor Detection using Image Processing", International Journal of Scientific Research in Computer Science, Engineering and Information Technology (IJSRCSEIT), ISSN : 2456-3307, Volume 7 Issue 3, pp. 348-352, May-June 2021. Available at doi : https://doi.org/10.32628/CSEIT217384 Journal URL : https://ijsrcseit.com/CSEIT217384 